\title{
MS11-02 | CCP4 Cloud for Distributed Crystallographic Computations
}

Krissinel, Eugene (Science \& Technology Facilities Council, Didcot, GBR); Lebedev, Andrey (STFC-UKRI, Didcot, GBR); Uski, Ville (Science \& Technology Facilities Council, Didcot, GBR); Ballard, Charles (Science \& Technology Facilities Council, Didcot, GBR); Keegan, Ronan (Science \& Technology Facilities Council, Didcot, GBR); Nicholls, Robert (MRC Laboratory of Molecular Biology, Cambridge, GBR); Kovalevskiy, Oleg (MRC Laboratory of Molecular Biology, Cambridge, GBR); Berrisford, John (European Bioinformatics Institute, Cambridge, GBR); Wojdyr, Marcin (Global Phasing Ltd, Cambridge, GBR); Simpkin, Adam (Institute of Integrative Biology, Liverpool, GBR); Thomas, Jens (Institute of Integrative Biology, Liverpool, GBR); Oliver, Christopher (Theoretical Physics Group, Birmingham, GBR); Pannu, Navraj (Biophysical Structural Chemistry, Leiden); Skubak, Pavol (Leiden University Medical Center, Leiden)

The Collaborative Computational Project Number 4 in Protein Crystallography (CCP4) exists to maintain, develop and provide world-class software that allows researchers to determine macromolecular structures by $\mathrm{X}$-ray crystallography and other biophysical techniques. Over 40 years of existence, CCP4 Software was assembled and distributed as an integrated Suite of programs, traditionally operated via CCP4i (2) GUI in Linux, OSX and Windows platforms.

Modern trends in computing suggest a fast-growing interest to mobile platforms and cloud solutions for data management and operations in practically all areas. Answering to these trends, CCP4 releases beta version of CCP4 Cloud, developed for essentially remote and distributed deployment of CCP4 Software. CCP4 Cloud allows a user to keep all necessary data and projects in the cloud and perform all scope of crystallographic computations, from image processing to final refinement, ligand fitting and deposition, remotely via a common web-browser, optionally complemented with CCP4 Cloud Client for interactive model building with Coot.

The talk will present architectural solutions and key features of CCP4 Cloud such as ability to seamlessly import data from 3rd party sites (e.g., synchrotrons), high scalability of computational background, convenient (big) data management for multiple users, rich graphical interface with built-in molecular graphics, enhanced data and structure solution pathway provenance.

CCP4 Cloud may be used from CCP4 Web portal with any device running a modern web-browser (including tablets and smartphones). All the source code is open and freely available for installation elsewhere to serve local researchers in a lab, or institution, or pharma, or a synchrotron. 